\title{
Alterations in MicroRNA Gene Expression Profile in Liver Transplant Patients with Hepatocellular Carcinoma
}

\section{Afsoon Afshari}

Shiraz University of Medical Sciences, Transplant Research center https://orcid.org/0000-0002-60863612

\section{Ramin Yaghobi}

Shiraz University of Medical Sciences

Mohammad hossin Karimi ( $\square$ karimimh@sums.ac.ir)

https://orcid.org/0000-0002-2435-6277

\section{Jvad Mowla}

Tarbiat Modares University Faculty of Biological Sciences

Research article

Keywords: LNA-Array, microRNA, Liver, Transplantation, Hepatocellular Carcinoma, qRT-PCR

Posted Date: August 20th, 2020

DOl: https://doi.org/10.21203/rs.3.rs-42044/v2

License: (c) (i) This work is licensed under a Creative Commons Attribution 4.0 International License. Read Full License 


\section{Abstract}

Background: HCC can lead to liver failure, rendering liver transplant. Since miRNAs can receive early evaluation before clinical symptoms are manifested, they might be detected as biomarkers of several disorders. Therefore, in the present study, alterations in miRNAs as biomarkers were detected in LT patients with HCC.

Methods: Fourteen tissue samples composed of 5 rejected and 9 non-rejected ones were used for studying the microRNAs expression pattern by LNA-array probe. The result was evaluated by qRT-PCR on 30 other tissue samples composed of 10 rejected and 20 non-rejected ones for the selected miRNAs. All samples were collected from liver transplanted patients with HCC.

Results: The study results revealed that in rejected patients compared to non-rejected ones, hsa-miR3158-5p, -4449, -4511, and -4633-5p were up-regulated and hsa-miR-122-3p, -194-5p, 548as-3p, and -4284 were down-regulated.

Conclusion: It was concluded that the tissue levels of specific miRNAs (especially hsa-miR-3158-5p, $-4449,-194-5 p$ and 548as-3p) correlated well with the development of HCC, which could be further studied as biomarkers.

\section{Background}

Many diseases might lead to liver failure for which the main treatment approach is liver transplant (LT) ${ }^{1}$. Ranked as the fifth most commonly diagnosed cancer worldwide, hepatocellular carcinoma (HCC) refers to a multistage process, involving many genes ${ }^{2}$, which can render to hepatocyte turnover, oxidative DNA damage, and inflammation ${ }^{3}$. Even though innovated therapeutic strategies are being established, handling advanced HCC is poorly efficacious and merely at the point of diagnosis. Apparently, transplantation seems to be the only known effective treatment approach for this disease ${ }^{4}$.

A considerable part of human genome is consisted of non-coding regions, including non-coding RNAs, known as microRNAs (miRNAs) ${ }^{1}$. These regions encode dynamic miRNAs, regulating around $20-30 \%$ of mammalian genes that comprise nearly $5 \%$ of transcriptome ${ }^{1,5}$. MiRNAs are evolutionary conserved, made up of 18-25 nucleotides. They are also involved in various biological processes e.g. cell growth, apoptosis, hematopoietic lineage differentiation, and gene regulation ${ }^{1,6,7}$.

There are also many miRNAs with specific expression patterns in the liver at different stages ${ }^{8}$. Investigations have demonstrated that some miRNAs are involved in regulating many metabolic pathways; hence, changes in their expression levels might reflect upon the underlying sources of inflammation ${ }^{9}$. The research which was conducted by Murakami et al., in 2006 was one of the pioneer studies on investigation the role of miRNAs in $\mathrm{HCC}$ development ${ }^{10}$. After that a number of studies have 
been investigated the miRNA changes in $\mathrm{HCC}^{11-15}$. As an illustration, it has been reported that miR-1792, miR-21, miR-221, miR-222, and miR-224 are frequently up-regulated in HCC. Furthermore, the importance of miRNAs in liver function has been broadly documented in the related literature ${ }^{16}$. Studies have also revealed that miR-122 as a blood marker changes in viral, alcoholic, and chemical injuries induced in the liver ${ }^{8}$. By the way, the importance of miRNAs is also, studied in LT rejection and it seems that some of miRNAs are playing specific role in this regard. Ectopic expression of serum miRNA signatures is known to be prognostically, diagnostically, and biologically related to liver allograft rejection

17. Other sources showed that in rat model of LT rejection, miR-146a, 15b, 223, 23a, 27a, 34a and 451 were upregulated ${ }^{18}$.

There are attempts to find miRNAs as early detectors and biomarkers. Nowadays, pathological tests being used to detect transplant outcomes mainly through biopsy, are invasive and take several days. Thus, the necessity for a faster and less invasive method seems crucial. Therefore, LNA-array (locked nucleic acid-array) probe and (quantitative) real-time polymerase chain reaction (qRT-PCR) were utilized to detect alterations in miRNA expression in the tissues of LT patients with HCC. The qRT-PCR was employed to certify the LNA-array probe results in the tissue samples of LT patients with HCC. This study is tried to elucidate the role of miRNAs in controlling the rejection of liver transplant in HCC patients.

\section{Methods}

\section{Methods}

\section{Patients and Samples}

This study was fulfilled in the Pathology Ward of Namazi Hospital, Shiraz, Iran (2016-2018). To conduct this study, a total number of 44 formalin-fixed paraffin-embedded (FFPE) samples were collected from LT patients with HCC. Macro-dissection was also performed. In brief, there were attempts to adopt a standard procedure to dissect the middle region of the tumor to harvest a proper sample, surrounded by the cancerous cells. The harvesting procedure was then confirmed microscopically to ensure the nonexistence of non-cancerous cells in each sample. All of the participants were asked to completed a questionnaire on demographic characteristics, history of other cancers except HCC, alcohol use, and tobacco use. Those with a history of other cancers, alcohol use, or tobacco use were excluded from the study. All of HCC patients' tumors were in stage III and IV. The sampling time of both acute rejected and nonrejected were the same.

The samples were divided into three groups, i.e., 14 samples used for LNA-array probe (the $1^{\text {st }}$ study group), 20 samples taken from non-rejected LT patients (the $2^{\text {nd }}$ study group), and the rest (the $3^{\text {rd }}$ group), 10 samples belonged to post-transplant samples from patients with acute rejection (Figure 1). All the protocols applied in this study were in complete agreement with the Helsinki Declaration and its later amendment. The local Ethics Committee of Shiraz University of Medical Sciences also approved each 
stage of this study. After explaining the study objectives, written informed consent was further obtained from each patient.

\section{De-Paraffinization of FFPE Samples, RNA Isolation and Quality Control}

Using a microtome, 10 cuts of $5 \mu \mathrm{m}$ of each sample were prepared. All the samples were RNA isolated, using Trizol ${ }^{\mathrm{TM}}$ (Invitrogen, Carlsbad, CA, USA) according to the manufacturer's protocol. Sample RNA Quality Control was further performed by an Agilent 2100 Bioanalyzer, to provide an electropherogram for each sample. In addition to the measurement of the rRNA ratio in a traditional manner (28S/18S), the Bioanalyzer provided an RNA Integrity Number (RIN) value (ranging from 0 to 10) and a reliable impression of RNA quality. The recommended RIN values were higher than 7 for good array performance. The NanoDrop instrument was also used to measure the concentration (A260), protein contamination (ratio A260/A280), and contamination with buffer components, or organic compounds (ratio A260/A230) accurately.

\section{Sample Preparation for LNA-Array Probe}

\section{Labeling, Hybridization, and Normalization}

Using the miRCURY LNA ${ }^{\mathrm{TM}}$ miRNA Hi-Power Labeling Kit (Exiqon, Denmark), 400ng total RNA were labeled by $\mathrm{Hy} 3^{\mathrm{TM}}$ and $\mathrm{Hy} 5^{\mathrm{TM}}$ fluorescent label for labeling the samples and the references, respectively to produce highly efficient and uniform labeling. The samples (labeled with $\mathrm{Hy}^{\mathrm{TM}}$ ) and the references (labeled with $\mathrm{Hy}^{\mathrm{TM}}$ ) were then mixed pairwise and recruited for hybridization. The hybridization procedure was performed using Tecan's HS $4800^{\text {TM }}$ hybridization station (Tecan, Austria) followed by miRCURY LNA ${ }^{\text {TM }}$ miRNA array instruction manual.

Later, the labeled specimens were hybridized by miRCURY LNA ${ }^{\mathrm{TM}}$ miRNA array $7^{\text {th }}$ Gen (Exiqon, Denmark) using the manufacturer's protocol. The quantified signals of the background were also corrected (Normexp with offset value 10) and normalized using the global Locally Weighted Scatterplot Smoothing (LOWESS) regression algorithm.

\section{Principle Component Analysis (PCA) Plot}

In order to decrease the proportions of the bulky data and to explore sample classes arising naturally and according to the expression profile, the PCA plot was applied. The top 50 miRNAs that had major variations across all the samples were also included.

\section{Array Slide Quality Control using Spike-Ins (Labeling Controls) and Normalization}

The spike-in controls were added in various concentrations in both $\mathrm{Hy} 3^{\mathrm{TM}}$ and $\mathrm{Hy} 5^{\mathrm{TM}}$ labeling reactions, giving the opportunity to evaluate the labeling reaction, hybridization, and performance of array experiment in a general manner (The level correlation of the signal intensities across all the slides is shown in the Additional File, Figure A1). 
The LOWESS also allows the correction of systematic deviations in the MA plot resulting in an intensitydependent adjustment of the MA-data to a straight line. The positive effect of this normalization is illustrated in the MA plots for each slide, showing the plot before and after normalization (see Additional File 1, Figure A2).

\section{qRT-PCR}

\section{Poly A Polymerization, cDNA Synthesis, and qRT-PCR}

To this end, $5 \mu \mathrm{g}$ of each isolated total RNAs was used for poly A polymerization by means of MiRNA cDNA Synthesis Kit (Parsgenome, Iran). Then, $2 \mu \mathrm{g}$ of the product was applied for the next step, i.e., cDNA synthesis using specific reverse primers for each miRNA. Finally, these miR specific cDNAs were employed as templates for quantitative SYBR Green Real-time analysis (Parsgenome, Iran). The expression level of the selected miRNA molecules was further determined employing SYBR Green Realtime PCR ( $\mathrm{ABI}, \mathrm{USA})$. In addition, U6 miRNA primers were included in the mentioned kit as an internal control.

The mix used for qRT-PCR for each reaction contained SYBR Green Premix $(10 \mu \mathrm{l}$ of Ex taq, Takara, Japan), Rox reference dye ( $0.4 \mu \mathrm{l})$, forward and reverse primers ( $1 \mu \mathrm{l}$ of $10 \mathrm{pM}$ mix of each primer pairs), and template $(2 \mu \mathrm{l}$ of synthesized cDNA, between 0.1 to $100 \mathrm{ng})$. Nuclease free water also added to this reaction up to $20 \mu \mathrm{l}$ total volume. The program employed for qRT-PCR was one cycle $95^{\circ} \mathrm{C}-5$ minutes, followed by 40 cycles of $95^{\circ} \mathrm{C}-5$ seconds, $61^{\circ} \mathrm{C}-20$ seconds, and $72^{\circ} \mathrm{C}-30$ seconds, followed by melting curve analysis for specificity of each reaction.

\section{Statistical Analysis}

The data were coded and imported into the IBM SPSS Statistics software (version 24). The quantitative variables were then compared using the non-parametric Kruskal-Wallis test for a comparison between more than two groups and the Mann-Whitney $U$ test was employed to compare two groups. The calculations for expression comparison in LNA-array tested samples were also executed by the R/Bioconductor software, using the LIMMA package. The $P$-value cut-off score for a significant difference was considered less than 0.05 .

\section{Results}

\section{Descriptive Statistics}

The $1^{\text {st }}$ study group was composed of 14 LT patients with the mean age of $47.4 \pm 7.8$ years (age range: 31 56) and included 9 (64.3\%) males. These samples were used for LNA-array probe. More data is shown in Figure 1 and Table 1 . The $2^{\text {nd }}$ study group consisted of 20 patients with no acute rejection of LT. Their mean age was also $49 \pm 15.3$ years (age range: $14-69)$ and included $14(70 \%)$ males. The $3^{\text {rd }}$ study group 
was composed of 10 patients showing acute rejection whose mean age was $49.7 \pm 17.1$ years (age range: 13-68) and included $6(75 \%)$ males.

\section{PCA Plot Analysis}

The top 50 miRNAs that had major variations across all the samples were used for PCA plot analysis. The results are depicted in Figure 2, demonstrating variations of the biological patterns or technical factors. Accordingly, PC1 and PC2 describe variations related to sample groups or treatments, whereas PC3 and later PC's might describe underlying or less variable factors like sample preparation conditions, operator, storage time, etc.

The unsupervised PCA and the hierarchical clustering could clearly show a tight grouping of the control samples (i.e., the non-rejected), whereas the treated samples (namely, the rejected) differed both from the controls as well as from each other. A simple expressed analysis by comparison did detect a subset of differentially expressed miRNAs including four up-regulated and four down-regulated miRNAs selected for further analyses.

\section{Heat Map and Unsupervised Hierarchical Clustering}

The heat map diagram shows the results of a two-way hierarchical clustering of miRNAs and the samples. The clustering was done using the complete-linkage method together with the Euclidean distance. A small subset of the miRNAs was also excluded from the heat map. As well, the normalized log ratio values were used for the analysis (Figure 3).

\section{MiRNA Expression Analysis for Rejected versus Non-Rejected in LNA-Arrayed Samples}

An expression analysis for the rejected cases versus the non-rejected ones was performed using a simple comparison method. The miRNA profiling accordingly identified a subset of top miRNAs (where the absolute value of the log fold change was larger than 1) out of the total number of miRNAs analyzed (371) by the miRCURY LNA ${ }^{T M}$ miRNA Array, differentially expressed in the rejected and the non-rejected samples. Table 2 provides a list of miRNAs with the most differential expression. The results showed that hsa-miR-122-3p, -548as-3p, -4284, and -194-5p had the highest levels of reduction amongst all the studied miRs, and hsa-miR-4511, $-4449,-4633-5 p$, and $-3158-5 p$ were the most increased ones; respectively.

\section{Selected miRNA Expression Level Comparison between Rejected and Non-Rejected Groups of Patients after LT}

The expression level of the selected miRs was tested in the other two groups of patients composed of rejected and non-rejected ones. The results of this selection are demonstrated in Figure 4A for the downregulated miRs and Figure 4B for the up-regulated ones based on a fold change. The results also revealed 
that hsa-miR-548as-3p and -194-5p had significantly reduced and hsa-miR-4449 and -3158-5p had shown a significant rise in the patients with LT rejection.

\section{Calculating Sensitivity and Specificity of Down-Regulated miRNAs between Non-Rejected and Rejected Groups of Patients after LT}

The sensitivity and the specificity of the down-regulated miRNAs between the non-rejected and rejected groups of patients were calculated (Figure 5). The area under curve (AUC), $p$-value, and $95 \%$ confidence interval $(95 \% \mathrm{Cl})$ of each down-regulated miRNA are demonstrated in Table 3. Two of the miRNAs (i.e. has-miR194-5p and -548as-3p) had a significant difference $(p<0.05)$, making them candidates as promising biomarkers.

\section{Calculating Sensitivity and Specificity of Up-Regulated miRNAs between Non-Rejected and Rejected Groups of Patients after LT}

The sensitivity and the specificity of the up-regulated miRNAs between the non-rejected and rejected groups of patients were calculated (Figure 6). The AUC, $p$-value, and $95 \% \mathrm{Cl}$ of each up-regulated miRNA are demonstrated in Table 4. Two of the miRNAs (namely, has-miR4449 and -3158-5p) established a significant difference $(p<0.05)$, making them candidates as promising biomarkers.

\section{Discussion}

The functions of miRNAs can provide valuable diagnostic measurements as well as prognostic indicators for several diseases including types of cancer ${ }^{5}$. A healthy liver consists of different cells producing a variety of miRNA expression profiles according to complex intrinsic and extrinsic signals. Under pathogenic conditions, these cells can also alter the profile of miRNAs ${ }^{19}$. Commonly, HCC is accompanied by loss of liver and the remaining treatment would be transplantation. Although many studied have been accomplished in order to find early detecting markers on the basis of miRNAs, still no effective biomarkers have been introduced in this regard (6 of 2019, Jin). By the way, as HCC renders to transplantation, the maintenance of the new liver and prevention from rejection is the priority of these patients. In the present research, it is tried to evaluate the alterations in miRNA expression profile of LT patients with HCC in order to introduce probable miRNA biomarker in early detection of rejection. In this sense, LNA-array probe was used to identify the expression profile of the miRNAs and the results of LNAarray was tested in other samples of liver transplanted patients with the same condition of underlying disease. Although it has been suggested that some of the circulating blood miRNAs have the potential to be used as miRNA-based blood biomarkers in cancer detection ${ }^{20,21}$, it is noteworthy to investigate the possibility of introducing more accurate miRNAs in detecting rejection in the biopsy samples that are taken commonly from patients. 
The findings revealed that miR-4449, miR-4511, miR-3158-5p, and miR-4633-5p had been up-regulated and miR-4284, miR-122-3p, miR-194-5p, and miR-548as-3p had been down-regulated in patients with LT rejection once compared with non-rejected ones. The results from LT patients were also in accordance with the LNA-array probe results. With reference to the results collected from the PCA plot, there was a significant difference between miRNA expression in the rejected LT patients and the non-rejected ones.

The miR-122-3p is a liver-specific miRNA, highly expressed, comprised of more than $70 \%$ of all cloned liver miRNAs, unlike other tissues ${ }^{1,22}$. Moreover, this miRNA plays a role in the liver metabolism, for instance, many genes interfering in lipid metabolism regulation can be down-regulated when using an antisense strategy to knock it down ${ }^{23}$. The sequence and the expression pattern of the miR-122-3p in the liver is also highly conserved ${ }^{24}$. In a study, HCC patients had shown a reduction in miR-122-3p expression 25. MiR-122-3p, miR-26a, and miR-195 have been also detected as tumor suppressors in the liver ${ }^{26}$. The fact that miR-122-3p reduces in HCC patients was accordingly certified in the present study. Other studies on liver transplant recipients with recurrent hepatitis $C$ and acute cellular rejection demonstrated the importance of miR-122 and miR-194 in liver transplant rejection either ${ }^{20,27}$. Furthermore, the correlation between miR122-3p and -194-5p has been a subject of research studies. Therefore, this miRNA was analyzed between HCC non-rejected and rejected patients, indicating that the given miRNA could significantly $(p=0.041)$ decrease in the rejected LT cases.

The miR-194-5p expression had been previously detected, reporting that such an miRNA could play a role in activation of stellate cells during liver fibrogenesis ${ }^{28,29}$. Another study had further profiled the expression of miR-194-5p as a marker of liver HCC metastasis by showing the overexpression of miR-194 in cancerous liver cell lines to down-regulate $\mathrm{N}$-cadherin expression and to suppress migration, invasion, and metastasis ${ }^{26}$. An increase in the expression of miR-194-5p in hepatitis C virus (HCV)-infected hepatoma cells has been also well documented although the miR-194-5p expression level had significantly reduced in HCC patients ${ }^{30}$. In addition, Shaker et al. had demonstrated that the expression levels of miR-122 could significantly correlate with miR-194 among HCC patients $(r=0.322, p=0.007)$. They had also reported no significant differences between non-rejected and rejected groups of patients against the expression of miR-194-5p ( $p=0.134)$. However, the difference in miR-122 expression level in their study was significant $(p=0.001)^{31}$. In addition, a research in 2015 had found that miR-194-5p had been down-regulated in the tissues involved in HCC and they had reported that it was able to reduce cell viability and proliferation by inducing G1 arrest and apoptosis in HCC cells. Moreover, they believed that miR-194-5p, inhibiting MAP4K4, was a potential diagnostic and therapeutic marker for HCC ${ }^{32}$.

Some miRNAs might be originated from repetitive elements like transposable elements (TEs) ${ }^{33}$. Hsa-mir548as-3p is also a member of a large human gene family, derived from transposable elements named Made ${ }^{34}$. Approximately 69 members are found in almost all human chromosomes, especially chromosomes 6, 8, and X (30.43\%). The predicted targets for hsa-miR-548as-3p are also functional molecules in many biological processes like MAPK signaling, cell cycle, p53 signaling pathway, colorectal cancer (CRC), non-small cell lung cancer, B cell receptor signaling pathway, transforming growth factor $\beta$ 
(TGF- $\beta$ ) signaling pathway, renal cell carcinoma, and many others ${ }^{35}$. TGF- $\beta$ signaling pathway is also important in post-transplant inflammation. Besides, it is considered as one of the main mediators and inducers of fibrosis. Therefore, miRNA down-regulation during the rejection period renders to overexpressed SMAD4, enhancing the signaling pathway of TGF- $\beta^{36}$. In addition, it has been reported that Tg737 is a target gene for miR-548as-3p, whose down-regulation facilitates HCC cell proliferation in vivo and in vitro, followed by an increase in colony formation ${ }^{37}$. The present study showed that the given miRNA had significantly $(p=0.041)$ reduced in rejected LT patients with HCC, accounting for its critical role in rejection.

The miRNA-4248 is located on chromosome 7, but there is not much data published about it. One study had used cross-mapping to detect miRNAs and had reported hsa-miR-4284 in cross-mapping events ${ }^{38}$. In addition, another research team had reported that such miRNAs could be the most down-regulated ones in the tissue samples of ulcerative colitis patients, and they had further claimed that miR-4284 level correlated with the disease activity by regulating CXCL5 mRNA expression ${ }^{39}$. Another research had found that miR-4284 and -4484 could be considered as diagnostic biomarkers for diffuse large B-cell lymphoma (DLBL) disease ${ }^{40}$. The down-regulation of this miRNA was further detected in LT patients with HCC in the present study. Furthermore, the sensitivity and the specificity of analyses of the four down-regulated miRNAs showed that miR194-5p and miR548as-3p might have enough qualities (Table 3 ) for being biomarker candidates for rejection especially among HCC patients.

In this research, a profile of miRNA was demonstrated in which miR-194-5p, -548as-3p, -122-3p, and -4284 were down-regulated and miR-3158-5p, $-4449,-4511$ and $-4633-5 p$ were upregulated in HCC patients with rejected LT. MiR-3158-5p is also located on chromosome 10 and has more than 500 predicted targets. The bioinformatic studies here showed that TGF- $\beta 2$ gene could be observed among its upper $95 \%$ target scores (see Additional File 1, Table A1; www.miRDB.org). TGF- $\beta$ also plays an important role in preparing a suitable microenvironment for tumor cell growth in liver diseases. This factor causes a signaling pathway that can promote progression of HCC in two ways; first, this factor has an intrinsic activity as an autocrine or paracrine growth factor, and second, it has extrinsic activity inducing changes in microenvironments like variations in cancer-associated fibroblasts, regulatory $T$ cells $\left(T_{\text {reg }}\right.$ cells), and inflammatory mediators ${ }^{41}$. The other up-regulated miRNA is miR-4449, located on chromosome 4 with 22 predicted targets in miRDB (see Additional File 1, Table A2). Among the predicted targets, the cyclindependent kinase 5 regulatory subunit 2, p39 (CDK5R2) is detected as the most highly scored one.

Previously, P39 had been introduced as a potential clinical prognostic marker for $\mathrm{HCC}^{42}$. Also, it had been detected that miR-4449 was a potential blood-based marker in multiple myeloma ${ }^{43}$. The present study suggested that these two mentioned miRNAs (i.e., miR-3158-5 $p$ and miR-4449) had significantly ( $p=0.014$ and 0.012 respectively) up-regulated in HCC patients with LT rejection. Even in analyzing sensitivity and specificity, these two miRNAs had AUC greater than 0.8 (refer to Table 4 for further information); therefore, they were proposed as a biomarker candidate for rejection in HCC patients. 
The third up-regulated miRNA in HCC patients with LT rejection was miR-4633-5p, located on chromosome 5 with more than 100 predicted targets in miRDB (see Additional File 1, Table A3). In 2015, a research group had further noted that miR-4633-5p could be significantly expressed in human epidermal growth factor receptor 2 (HER2)-positive breast carcinomas among 85 tested miRNAs versus normal people ${ }^{44}$. The last up-regulated studied miRNA in LT rejected patients with HCC is miR-4511, located on chromosome 15 with more than 400 predicted targets in miRDB (see Additional File 1, Table A4). There are also few studies on the role of miRNA in LT and further studies are warranted to detect the exact role of this miR and its targets. The results of the present study showed the up-regulation of these two miRNAs in HCC patients with rejected LT.

It is of note that more than $97 \%$ of liver biopsies are hepatocytes and biliary cells, and the rest are Kupffer cells and endothelial ones. In LT rejected biopsies, some macrophages, plasma cells, and lymphocytes are also added to their population ${ }^{45}$. Given the fact that the selected miRNAs are expressed in the nonrejected group of patients and the majority of cells in biopsies are hepatocytes, it can be concluded that alterations in the expression profile of the selected miRs might be due to the rejection process in hepatocytes.

\section{Conclusion}

Even though the biological function of miRNAs is yet to be fully understood, it has been shown that the tissue levels of specific miRNAs correlate well with the pathological development of several different types of cancer. Previously, it has been suggested that some blood miRNA have the potential to be used as miRNA-based blood biomarkers in cancer detection. In summary, the identification of miRNAs and small RNA species seems to represent only the tip of the iceberg. In addition, the prediction of an individual miRNA, its target, and even its function is still one of the biggest challenges in research. In the present study, two down-regulated (i.e. miR194-5p and miR584as-3p) and two up-regulated (namely, miR4449 and miR-3158-5p) miRNAs were introduced as candidate biomarkers for LT rejection in HCC patients which need to be confirmed by further studies.

\section{Abbreviations}

alanine transaminase (ALT)

aspartate transaminase (AST)

a-fetoprotein (AFT)

Hepatocellular carcinoma (HCC)

microRNAs (miRNAs) 
3'-untranslated regions (3'-UTRs)

LNA-array (locked nucleic acid-array)

formalin-fixed paraffin-embedded (FFPE)

transposable elements (TEs)

Transforming growth factor $\beta$ (TGF- $\beta$ )

large B-cell lymphoma (DLBL)

\section{Declarations}

\section{Ethics approval and consent to participate:}

All applied protocols in this study, were in complete agreement with the Helsinki Declaration and its later amendment. The local Ethics committee of Shiraz University of Medical Sciences approved every step of this study. After explaining the study objectives, written informed consent was obtained from each patient.

Consent for publication:

written informed consent for publication was obtained.

Availability of data and material:

The datasets used and/or analyzed during the current study are available from the corresponding author on reasonable request.

Competing interests:

The authors declare that they have no competing interests.

\section{Funding:}

This work was supported by grants from Transplant Research Center, Shiraz University of Medical Sciences, with grant No. 91/181. Funding sources had no influence over study design, data collection, analysis or interpretation or manuscript preparation and submission.

\section{Authors' contributions:}


AA performed the majority of experiments and analyzed the data; AA, KM, YR performed the molecular investigations; AA, KM, YR and MJ designed and coordinated the research; AA, KM, YR and MJ wrote the paper. AA and YR have equal role as first author.

\section{Acknowledgements:}

The authors wish to thank Mr. H. Argasi at the Research Consultation Center (RCC) of Shiraz University of Medical Sciences for his invaluable assistance in editing this manuscript.

\section{References}

1. Chen XM. MicroRNA signatures in liver diseases. World J Gastroenterol. 2009;15(14):1665-1672. doi:10.3748/wjg.15.1665

2. Huang S, He X. The role of microRNAs in liver cancer progression. Br J Cancer. 2011;104(2):235-240. doi:10.1038/sj.bjc.6606010

3. Dragani TA. Risk of HCC: Genetic heterogeneity and complex genetics. J Hepatol. 2010;52(2):252257. doi:10.1016/j.jhep.2009.11.015

4. Jemal A, Center MM, DeSantis C, Ward EM. Global Patterns of Cancer Incidence and Mortality Rates and Trends. Cancer Epidemiol Biomarkers Prev. 2010;19(8):1893-1907. doi:10.1158/1055-9965.EPI10-0437

5. Castoldi M, Schmidt S, Benes V, et al. A sensitive array for microRNA expression profiling (miChip ) based on locked nucleic acids ( LNA ) A sensitive array for microRNA expression profiling (miChip ) based on locked nucleic acids ( LNA ). RNA Soc. 2006;12:913-920. doi:10.1261/rna.2332406.a

6. Barad O, Meiri E, Avniel A, et al. MicroRNA expression detected by oligonucleotide microarrays: System establishment and expression profiling in human tissues. Methods. 2004;14:2486-2494. doi:10.1101/gr.2845604.4

7. Song Q, Song J, Wang Q, et al. miR-548d-3p/TP53BP2 axis regulates the proliferation and apoptosis of breast cancer cells. Cancer Med. 2016;5(2):315-324. doi:10.1002/cam4.567

8. Lewis AP, Jopling CL. Regulation and biological function of the liver-specific miR-122. Biochem Soc Trans. 2010;38(6):1553-1557. doi:10.1042/BST0381553

9. Hayes C, Chayama K. MicroRNAs as Biomarkers for Liver Disease and Hepatocellular Carcinoma. Int J Mol Sci. 2016;17(3):280. doi:10.3390/ijms17030280

10. Murakami Y, Yasuda T, Saigo K, et al. Comprehensive analysis of microRNA expression patterns in hepatocellular carcinoma and non-tumorous tissues. Oncogene. 2006;25(17):2537-2545. doi:10.1038/sj.onc.1209283

11. Yang N, Ekanem NR, Sakyi CA, Ray SD. Hepatocellular carcinoma and microRNA: New perspectives on therapeutics and diagnostics. Adv Drug Deliv Rev. 2015;81:62-74. doi:10.1016/j.addr.2014.10.029 
12. Gramantieri L, Ferracin M, Fornari $F$, et al. Cyclin $G 1$ is a target of miR-122a, a MicroRNA frequently down-regulated in human hepatocellular carcinoma. Cancer Res. 2007;67(13):6092-6099. doi:10.1158/0008-5472.CAN-06-4607

13. Burchard J, Zhang C, Liu AM, et al. MicroRNA-122 as a regulator of mitochondrial metabolic gene network in hepatocellular carcinoma. Mol Syst Biol. 2010;6:402. doi:10.1038/msb.2010.58

14. Mizuguchi Y, Mishima T, Yokomuro $S$, et al. Sequencing and Bioinformatics-Based Analyses of the microRNA Transcriptome in Hepatitis B-Related Hepatocellular Carcinoma. Guan X, ed. PLoS One. 2011;6(1):e15304. doi:10.1371/journal.pone.0015304

15. Wang Y, Lee ATC, Ma JZI, et al. Profiling microRNA expression in hepatocellular carcinoma reveals microRNA-224 up-regulation and apoptosis inhibitor-5 as a microRNA-224-specific target. $J$ Biol Chem. 2008;283(19):13205-13215. doi:10.1074/jbc.M707629200

16. Sui W, Lin H, Peng W, et al. Molecular dysfunctions in acute rejection after renal transplantation revealed by integrated analysis of transcription factor, microRNA and long noncoding RNA. Genomics. 2013;102(4):310-322. doi:10.1016/j.ygeno.2013.05.002

17. Shaked A, Chang BL, Barnes MR, et al. An ectopically expressed serum miRNA signature is prognostic, diagnostic, and biologically related to liver allograft rejection. Hepatology. 2017;65(1):269-280. doi:10.1002/hep.28786

18. Morita $M$, Chen J, Fujino $M$, et al. Identification of microRNAs involved in acute rejection and spontaneous tolerance in murine hepatic allografts. Sci Rep. 2014;4. doi:10.1038/srep06649

19. Chen X-M, Splinter PL, O'Hara SP, LaRusso NF. A cellular micro-RNA, let-7i, regulates Toll-like receptor 4 expression and contributes to cholangiocyte immune responses against Cryptosporidium parvum infection. J Biol Chem. 2007;282(39):28929-28938. doi:10.1074/jbc.M702633200

20. Asaoka T, Hernandez D, Tryphonopoulos $P$, et al. Clinical significance of intragraft miR-122 and -155 expression after liver transplantation. Hepatol Res. 2015;45(8):898-905. doi:10.1111/hepr.12424

21. Jin Y, Wong YS, Goh BKP, et al. Circulating microRNAs as Potential Diagnostic and Prognostic Biomarkers in Hepatocellular Carcinoma. Sci Rep. 2019;9(1):1-12. doi:10.1038/s41598-019-46872-8

22. Chang J, Nicolas E, Marks $D$, et al. miR-122, a mammalian liver-specific microRNA, is processed from hcr mRNA and may downregulate the high affinity cationic amino acid transporter CAT-1. RNA Biol. 2004;1(2):106-113.

23. Arora A, Simpson DA. Individual mRNA expression profiles reveal the effects of specific microRNAs. Genome Biol. 2008;9(5):R82. doi:10.1186/gb-2008-9-5-r82

24. Wienholds E, Kloosterman WP, Miska E, et al. MicroRNA Expression in Zebrafish Embryonic Development. Science (80-). 2005;309(5732):310-311. doi:10.1126/science.1114519

25. Coulouarn C, Factor VM, Andersen JB, Durkin ME, Thorgeirsson SS. Loss of miR-122 expression in liver cancer correlates with suppression of the hepatic phenotype and gain of metastatic properties. Oncogene. 2009;28(40):3526-3536. doi:10.1038/onc.2009.211

26. Meng Z, Fu X, Chen X, et al. miR-194 is a marker of hepatic epithelial cells and suppresses metastasis of liver cancer cells in mice. Hepatology. 2010;52(6):2148-2157. doi:10.1002/hep.23915 
27. Farid WRR, Pan Q, van der Meer AJP, et al. Hepatocyte-derived microRNAs as serum biomarkers of hepatic injury and rejection after liver transplantation. Liver Transpl. 2012;18(3):290-297. doi:10.1002/lt.22438

28. Raymond CK, Roberts BS, Garrett-Engele P, Lim LP, Johnson JM. Simple, quantitative primerextension PCR assay for direct monitoring of microRNAs and short-interfering RNAs. RNA. 2005;11(11):1737-1744. doi:10.1261/rna.2148705

29. Venugopal SK, Jiang J, Kim T-H, et al. Liver fibrosis causes downregulation of miRNA-150 and miRNA-194 in hepatic stellate cells, and their overexpression causes decreased stellate cell activation. Am J Physiol Gastrointest Liver Physiol. 2010;298(1):G101-6. doi:10.1152/ajpgi.00220.2009

30. Jung HS, Seo Y-R, Yang YM, et al. Ga12gep oncogene inhibits FOXO1 in hepatocellular carcinoma as a consequence of miR-135b and miR-194 dysregulation. Cell Signal. 2014;26(7):1456-1465. doi:10.1016/j.cellsig.2014.02.022

31. Shaker OG, El-shehaby AR, Zahra AMRA, El-aleem OOABD, Yousry A. Hepatocyte-Derived MicroRNAS as Serum Predictors of Rejection of Liver Transplantation. 2015;83(2):103-108.

32. Zhao Y, Li F, Zhang $X$, et al. MicroRNA-194 acts as a prognostic marker and inhibits proliferation in hepatocellular carcinoma by targeting MAP4K4. Int J Clin Exp Pathol. 2015;8(10):12446-12454.

33. Yuan Z, Sun X, Liu H, Xie J. MicroRNA Genes Derived from Repetitive Elements and Expanded by Segmental Duplication Events in Mammalian Genomes. Tjwa M, ed. PLoS One. 2011;6(3):e17666. doi:10.1371/journal.pone.0017666

34. Piriyapongsa J, Jordan IK. A family of human microRNA genes from miniature inverted-repeat transposable elements. Christoffels A, ed. PLoS One. 2007;2(2):e203. doi:10.1371/journal.pone.0000203

35. Liang T, Guo L, Liu C. Genome-Wide Analysis of mir-548 Gene Family Reveals Evolutionary and Functional Implications. J Biomed Biotechnol. 2012;2012:1-8. doi:10.1155/2012/679563

36. Hamdorf M, Kawakita S, Everly M. The Potential of MicroRNAs as Novel Biomarkers for Transplant Rejection. J Immunol Res. 2017;2017:1-12. doi:10.1155/2017/4072364

37. Zhao G, Wang T, Huang Q-K, et al. MicroRNA-548a-5p promotes proliferation and inhibits apoptosis in hepatocellular carcinoma cells by targeting Tg737. World J Gastroenterol. 2016;22(23):5364. doi:10.3748/wjg.v22.i23.5364

38. Guo L, Liang T, Gu W, Xu Y, Bai Y, Lu Z. Cross-mapping events in miRNAs reveal potential miRNAMimics and evolutionary implications. PLoS One. 2011;6(5). doi:10.1371/journal.pone.0020517

39. Koukos G, Polytarchou C, Kaplan JL, et al. A MicroRNA Signature in Pediatric Ulcerative Colitis. Inflamm Bowel Dis. 2015;21(5):996-1005. doi:10.1097/MIB.0000000000000339

40. Tamaddon G, Geramizadeh B, Karimi MH, Mowla SJ, Abroun S. miR-4284 and miR-4484 as Putative Biomarkers for Diffuse Large B-Cell Lymphoma. Iran J Med Sci. 2016;41(4):334-339.

41. Giannelli G, Villa E, Lahn M. Transforming growth factor- $\beta$ as a therapeutic target in hepatocellular carcinoma. Cancer Res. 2014;74(7):1890-1894. doi:10.1158/0008-5472.CAN-14-0243 
42. Lu J-W, Chang J-G, Yeh K-T, Chen R-M, Tsai JJP, Hu R-M. Decreased expression of p39 is associated with a poor prognosis in human hepatocellular carcinoma. Med Oncol. 2011;28(S1):239-245. doi:10.1007/s12032-010-9707-9

43. Shen X, Ye Y, Qi J, et al. Identification of a novel microRNA, miR-4449, as a potential blood based marker in multiple myeloma. Clin Chem Lab Med. 2017;55(5):748-754. doi:10.1515/cclm-2015-1108

44. Vos S, Vesuna F, Raman V, van Diest PJ, van der Groep P. miRNA expression patterns in normal breast tissue and invasive breast cancers of BRCA1 and BRCA2 germ-line mutation carriers. Oncotarget. 2015;6(31):32115-32137. doi:10.18632/oncotarget.5617

45. Ross MH, Kaye GI, Pawlina W. Histology: A Text and Atlas with Cell and Molecular Biology. Lippincott Williams \& Wilkins; 2003.

\section{Tables}

Table 1: Descriptive statistics related to first group of patients

\begin{tabular}{|c|c|c|c|c|c|}
\hline & \multicolumn{2}{|c|}{ Rejection condition } & \multirow[b]{2}{*}{ Total } \\
\hline & & & Rejected & Non-Rejected & \\
\hline \multirow[t]{6}{*}{ Gender } & Male & Count & 3 & 6 & 9 \\
\hline & & $\%$ within gender & $33.3 \%$ & $66.7 \%$ & $100.0 \%$ \\
\hline & & $\%$ within rejection & $60.0 \%$ & $66.7 \%$ & $64.3 \%$ \\
\hline & Female & Count & 2 & 3 & 5 \\
\hline & & $\%$ within gender & $40.0 \%$ & $60.0 \%$ & $100.0 \%$ \\
\hline & & $\%$ within rejection & $40.0 \%$ & $33.3 \%$ & $35.7 \%$ \\
\hline \multirow[t]{3}{*}{ Total } & & Count & 5 & 9 & 14 \\
\hline & & $\%$ within gender & $35.7 \%$ & $64.3 \%$ & $100.0 \%$ \\
\hline & & $\%$ within rejection & $100.0 \%$ & $100.0 \%$ & $100.0 \%$ \\
\hline
\end{tabular}


Table 2: The table shows the selected differentially expressed microRNA candidates, ranked according to the absolute value of the log fold change

\begin{tabular}{|lllll|}
\hline Annotation & Average Hy3 & $\begin{array}{l}\text { Non-reject } \\
\text { fold change }\end{array}$ & $\begin{array}{l}\text { Reject } \\
\text { fold change }\end{array}$ & Log FC \\
\hline hsa-miR-122-3p & 9.025 & 1.068 & -1.013 & -0.793 \\
\hline hsa-miR-194-5p & 9.161 & 0.606 & -1.051 & -0.258 \\
\hline hsa-miR-548as-3p & 8.661 & 0.015 & -1.714 & -1.326 \\
\hline hsa-miR-4284 & 13.779 & 0.893 & -1.069 & -0.759 \\
\hline hsa-miR-3158-5p & 8.303 & -1.651 & 0.745 & 0.873 \\
\hline hsa-miR-4449 & 7.259 & -1.399 & 0.935 & 1.035 \\
\hline hsa-miR-4511 & 7.337 & -1.656 & 0.775 & 1.204 \\
\hline hsa-miR-4633-5p & 8.176 & -1.704 & 0.658 & 0.973 \\
\hline
\end{tabular}

Table 3: Sensitivity and specificity of down-regulated microRNAs between non-rejected and rejected groups of patients after transplantation

\begin{tabular}{|llll|}
\hline miRNA & Area under the curve (AUC) & $p$ value & $95 \% \mathrm{Cl}$ \\
\hline has-miR122-3p & 0.7083 & 0.1228 & $0.4740-0.9427$ \\
\hline has-miR4284 & 0.5228 & 0.8688 & $0.2506-0.7949$ \\
\hline has-miR194-5p & 0.7987 & 0.0372 & $0.5414-1.000$ \\
\hline has-miR548as-3p & 0.8250 & 0.0344 & $0.645-1.000$ \\
\hline
\end{tabular}

Table 4: Sensitivity and specificity of up-regulated microRNAs between non-rejected and rejected groups of patients after transplantation

\begin{tabular}{|llll|}
\hline miRNA & Area under the curve (AUC) & $p$ value & $95 \% \mathrm{Cl}$ \\
\hline has-miR4511 & 0.6000 & 0.5152 & $0.3134-0.8866$ \\
\hline has-miR4633-5p & 0.7424 & 0.1078 & $0.4985-0.9864$ \\
\hline has-miR4449 & 0.8385 & 0.0122 & $0.6620-1.000$ \\
\hline has-miR3158-5p & 0.8561 & 0.0182 & $0.5946-1.000$ \\
\hline
\end{tabular}


Figures

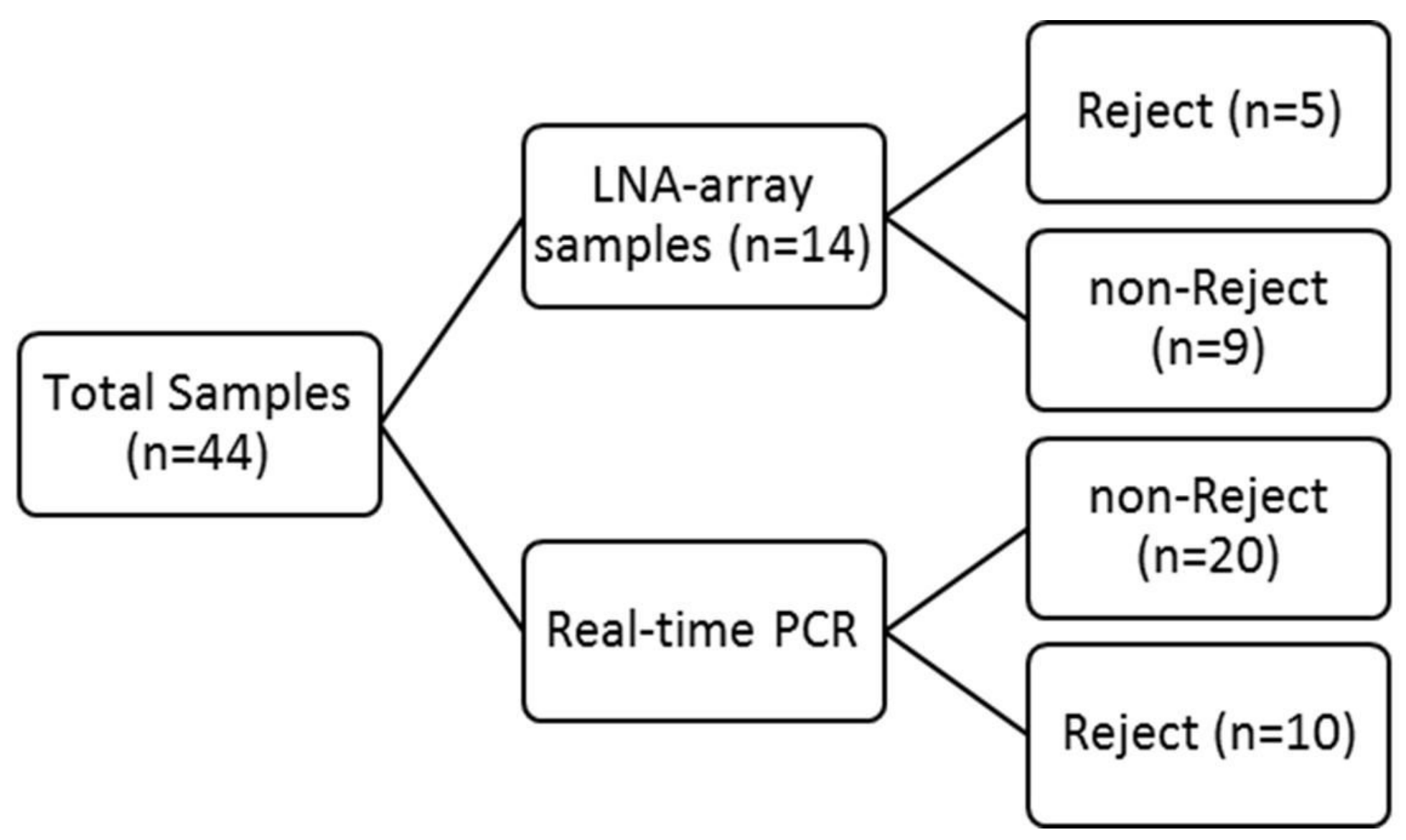

Figure 1

The study sample division chart. 


\section{Color Key}

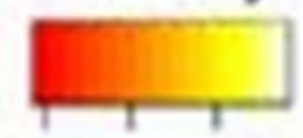

$-50.5$

Value

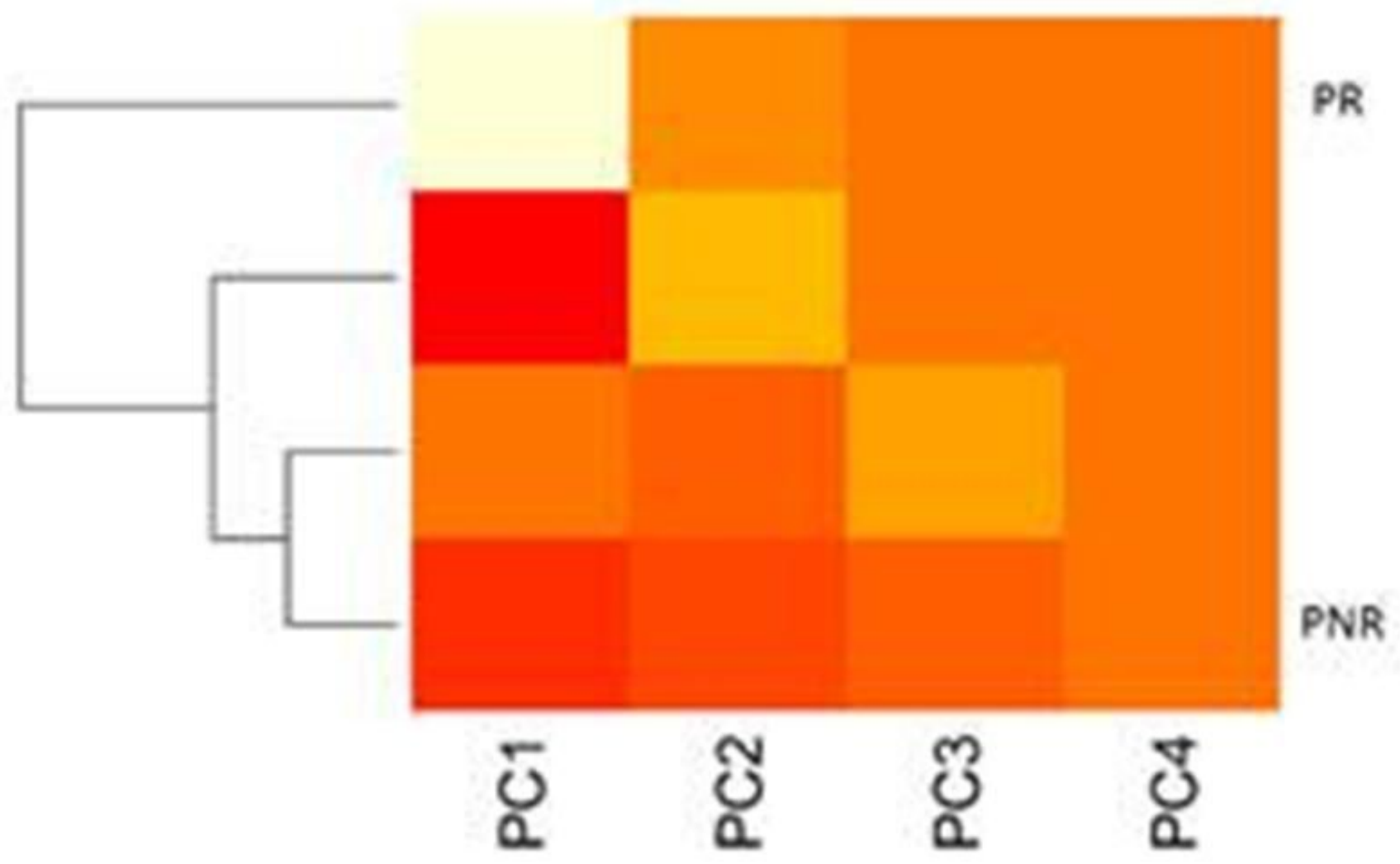

Figure 2

Matrix PCA plot. Analysis was performed on all samples. The normalized log ratio values were used for the analysis. Here, PR means pool of reject samples while PNR means pool of non-reject samples. 


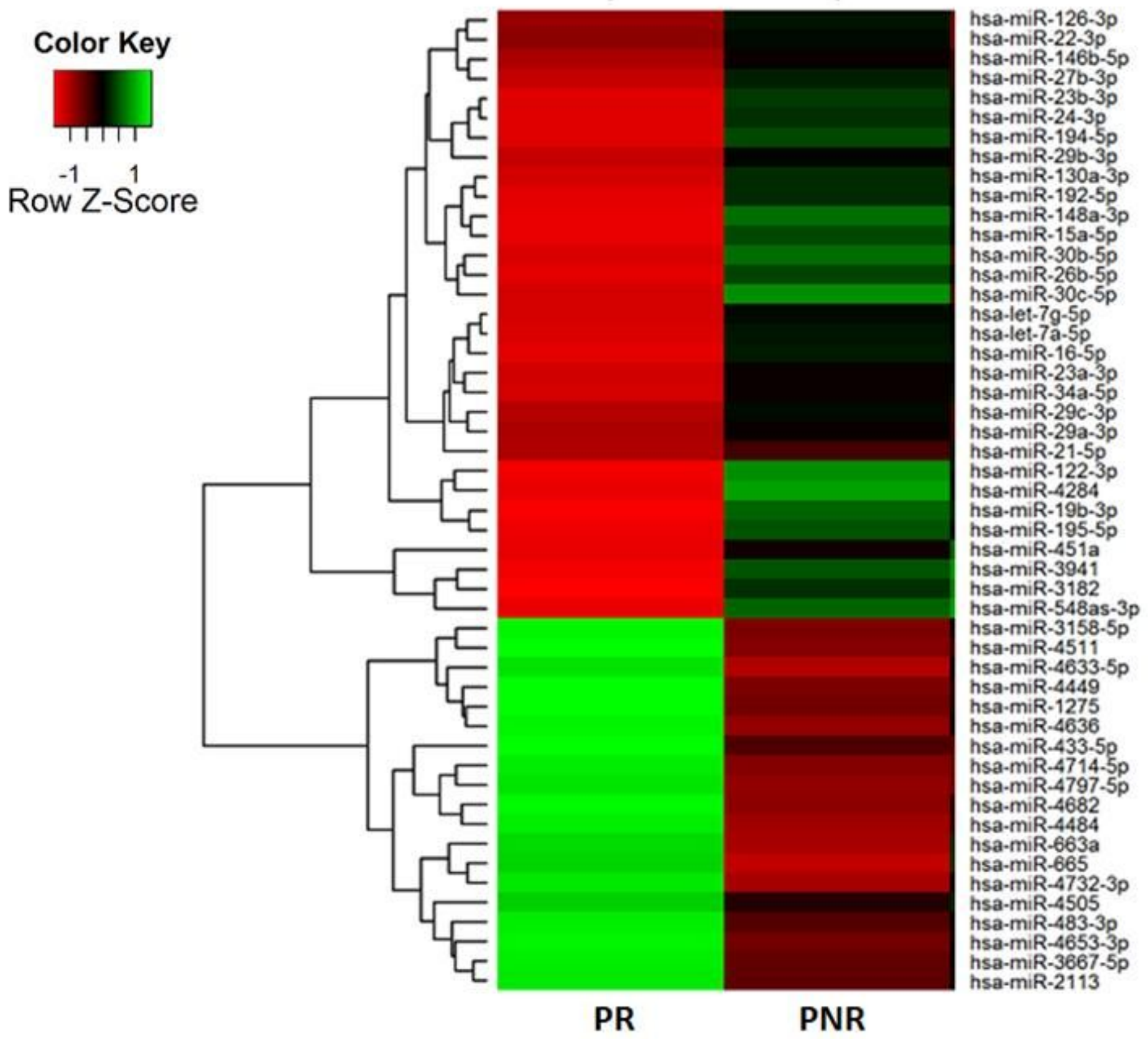

\section{Figure 3}

Each row represents a microRNA and each column represents a sample. The color scale illustrates the relative expression level of microRNAs. Red color represents an expression level below the reference channel, and green color represents expression higher than the reference. 

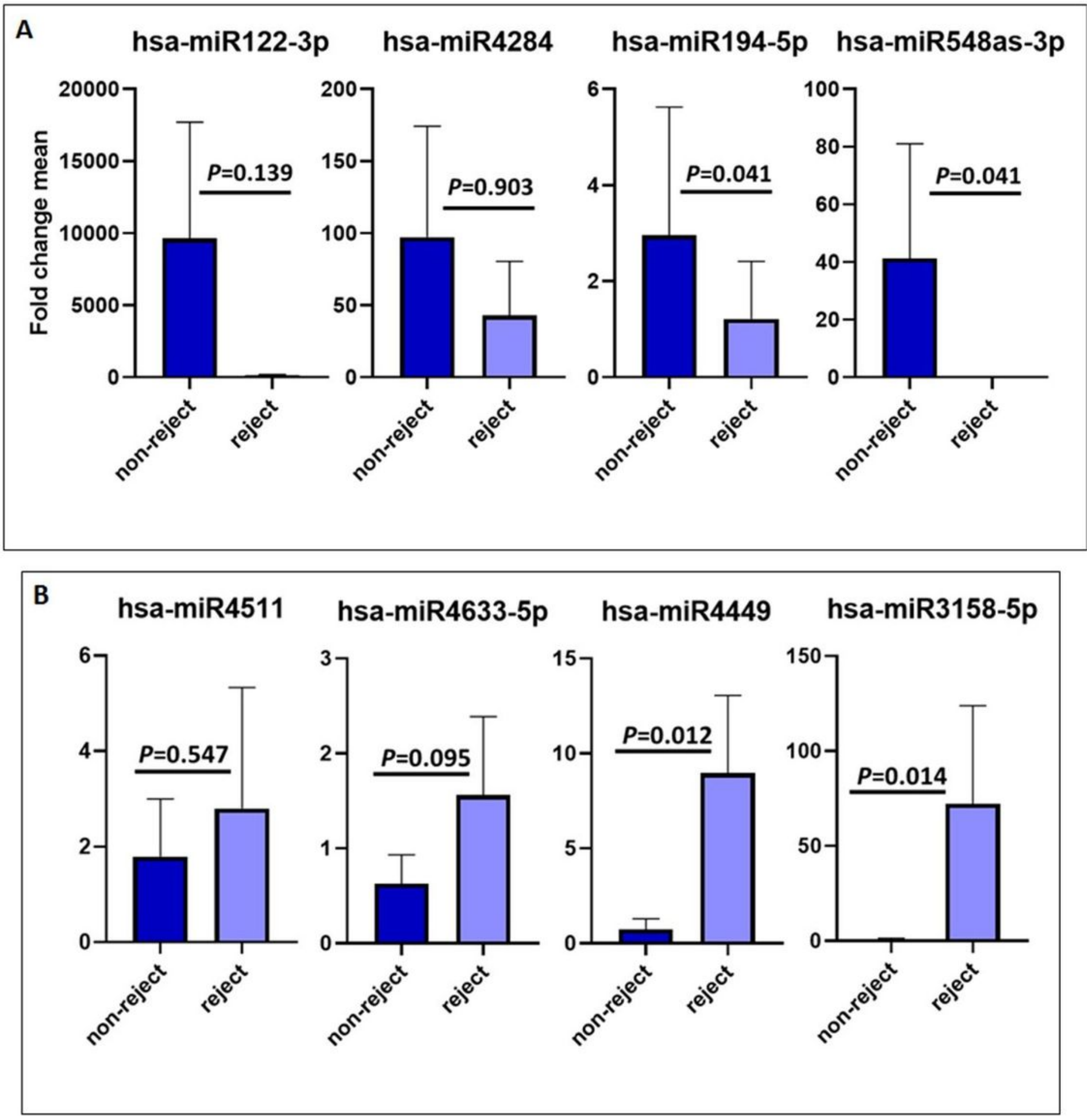

Figure 4

The expression level of selected miRs for down regulated miRs (A) and for up regulated ones (B) based on fold change. 
ROC curve: ROC of hsa-miR122-3p

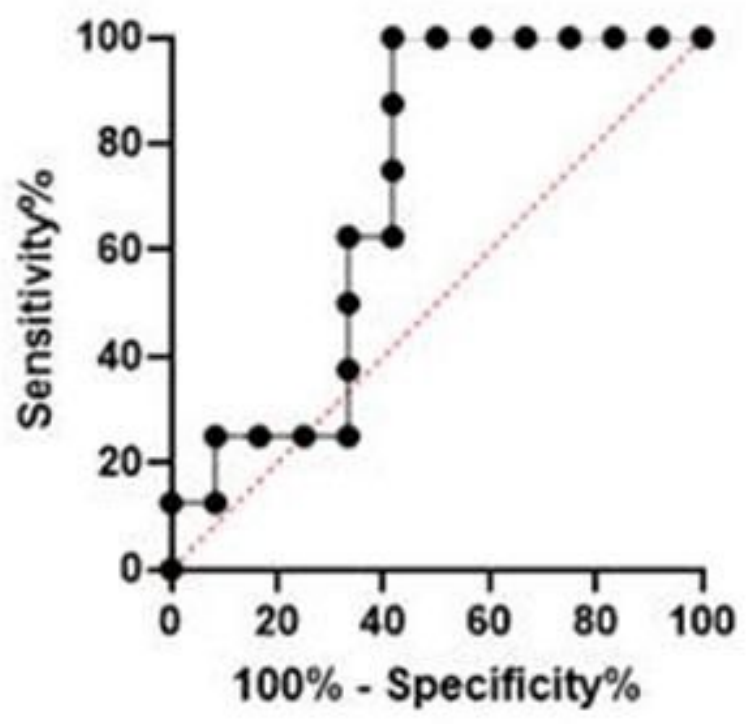

ROC curve: ROC of hsa-miR4284

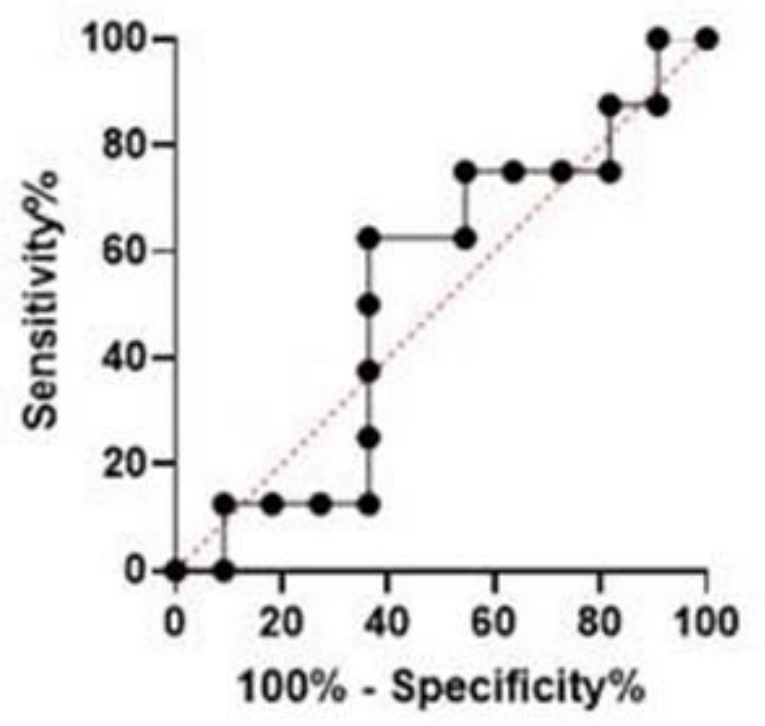

ROC curve: ROC of hsa-miR194-5p ROC curve: ROC of hsa-miR548as-3p
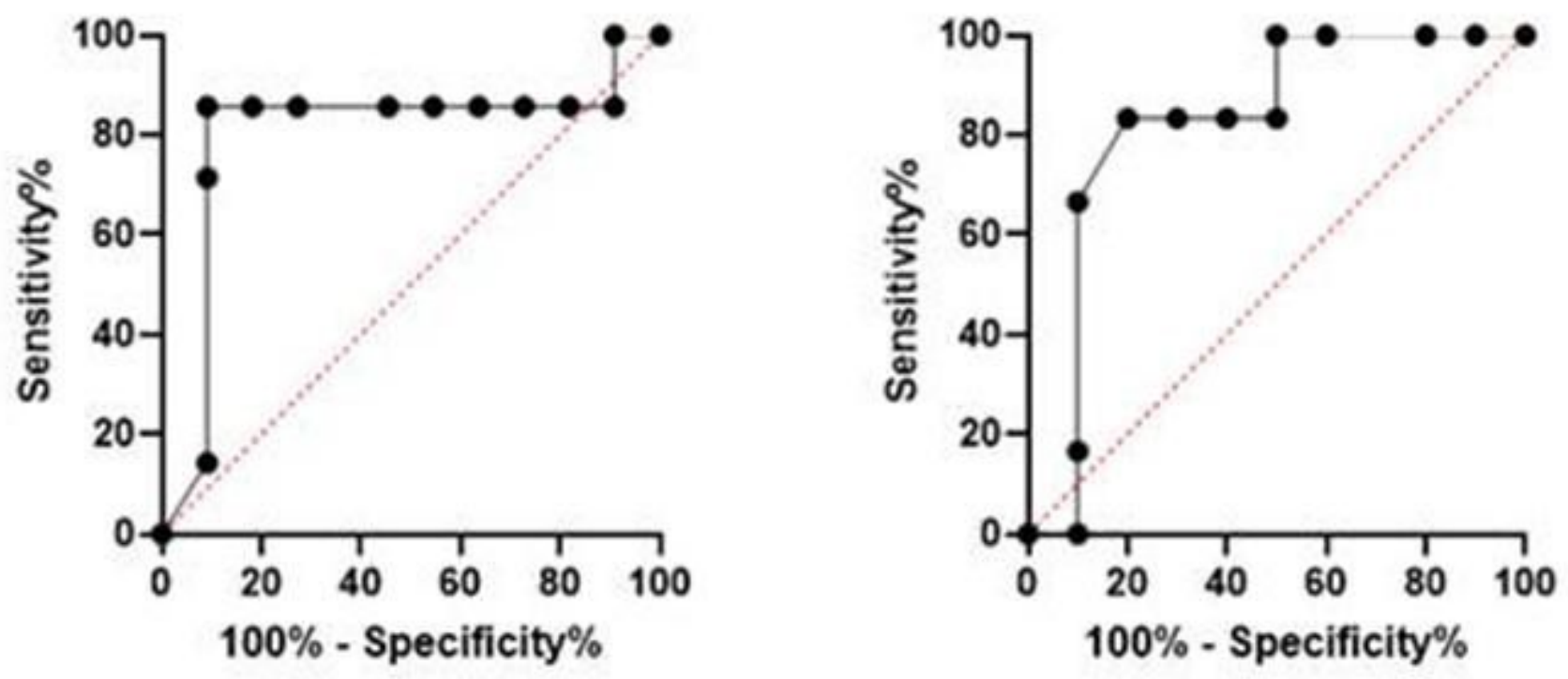

Figure 5

Calculating the sensitivity and specificity of down-regulated microRNAs between non-rejected and rejected groups of patients after transplantation. 
ROC curve: ROC of hsa-miR4511

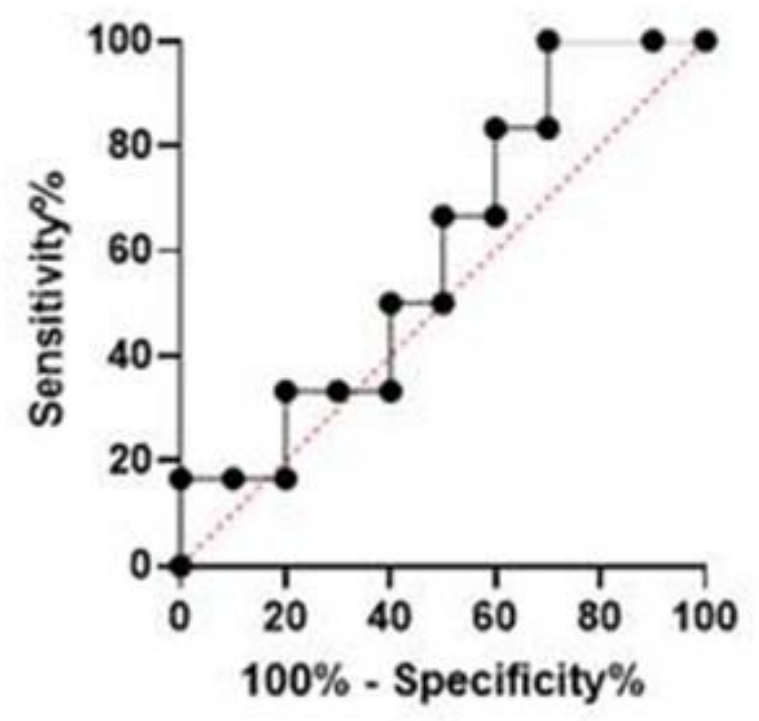

ROC curve: ROC of hsa-miR4449

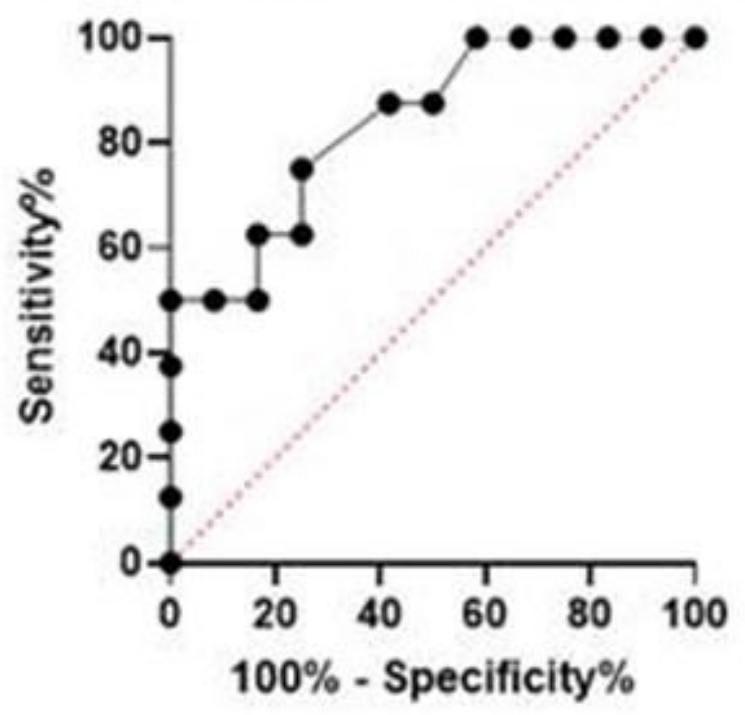

ROC curve: ROC of hsa-miR4633-5p

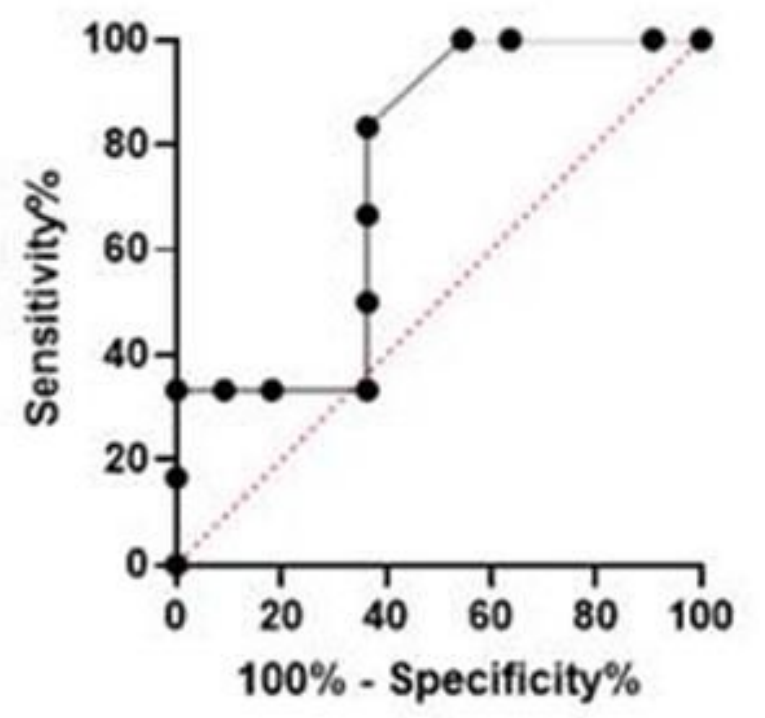

ROC curve: ROC of hsa-miR3158-5p

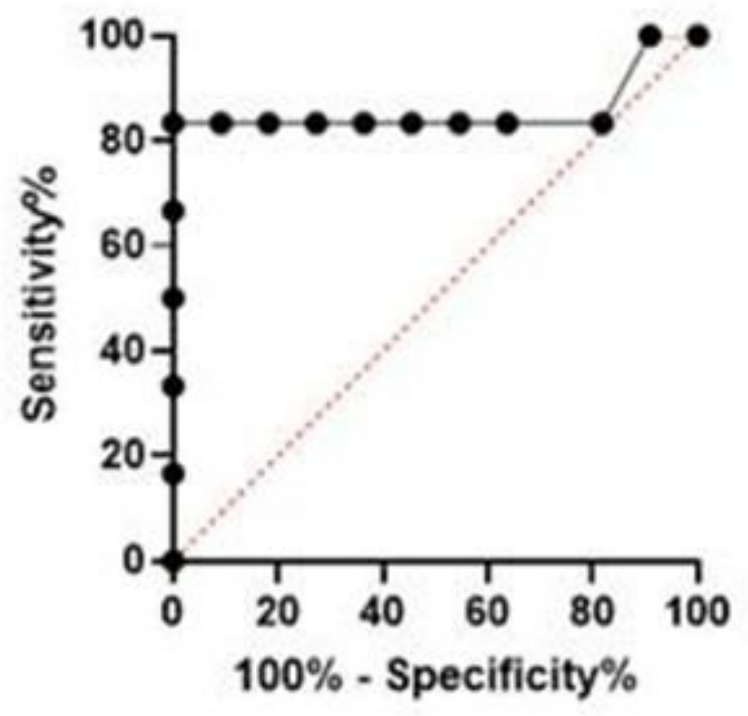

Figure 6

Calculating the sensitivity and specificity of up-regulated microRNAs between non-rejected and rejected groups of patients after transplantation.

\section{Supplementary Files}

This is a list of supplementary files associated with this preprint. Click to download.

- Additionalfile.docx 